REVUE DE L'INSTITUT

FRANÇAIS D'HISTOIRE

EN ALLEMAGNE

\section{Revue de l'IFHA}

Revue de l'Institut français d'histoire en Allemagne

$1 \mid 2009$

IFHA 1

\title{
Tr@jectoires. Travaux des jeunes chercheurs du CIERA
}

\section{Falk Bretschneider}

\section{OpenEdition}

\section{Journals}

Édition électronique

URL : http://journals.openedition.org/ifha/348

DOI : 10.4000/ifha.348

ISSN : 2198-8943

\section{Éditeur}

IFRA - Institut franco-allemand (sciences historiques et sociales)

\section{Édition imprimée}

Date de publication : 30 septembre 2009

Pagination : 162-166

ISSN : 2190-0078

\section{Référence électronique}

Falk Bretschneider, «Tr@jectoires. Travaux des jeunes chercheurs du CIERA»,Revue de l'IFHA [En ligne],

1 | 2009, mis en ligne le 07 février 2013, consulté le 25 avril 2019. URL : http://

journals.openedition.org/ifha/348 ; DOI : 10.4000/ifha.348

Ce document a été généré automatiquement le 25 avril 2019.

(CIFHA 


\title{
Tr@jectoires. Travaux des jeunes chercheurs du CIERA
}

\author{
Falk Bretschneider
}

1 Ouvrir un forum à la jeune recherche franco-allemande en sciences humaines et sociales : telle est l'ambition de la revue Tr@jectoires. Travaux des jeunes chercheurs du CIERA. Fondée, en 2007, par une équipe de doctorants et post-doctorants du Centre interdisciplinaire d'études et de recherches sur l'Allemagne, ${ }^{1}$ cette jeune revue annuelle, bilingue (les contributions sont publiées en français ou en allemand) et résolument interdisciplinaire est née d'une triple volonté : porter un regard synthétique et transversal sur des recherches en cours, participer à l'émergence d'une nouvelle génération de jeunes chercheurs travaillant sur le monde germanique et contribuer à faire valoir les travaux des inscrits du CIERA (à l'heure actuelle environ 700 étudiants, doctorants et postdoctorants) auprès de la communauté scientifique en France et en Allemagne. Issu d'une réflexion autour des nouvelles formes possibles de la formation à la recherche, le projet s'inscrit pleinement dans les missions du CIERA qui sont la promotion des formations doctorales et de la recherche française sur l'Allemagne, le dépassement des frontières entre les disciplines et l'intensification des collaborations scientifiques entre étudiants, enseignants et jeunes chercheurs de part et d'autre du Rhin. La revue complète ainsi d'autres dispositifs offerts par ce Centre pour renforcer l'intégration des jeunes chercheurs dans la communauté scientifique des études interdisciplinaires francoallemandes - mentionnons notamment le programme d'encadrement doctoral proposé, depuis la rentrée 2006, pour mettre à la disposition des doctorants une structure adaptée à leurs besoins en termes d'information, de formation, de soutien méthodologique et de visibilité.

\section{Fonctionnement de la revue}

2 L'originalité de Tr@jectoires repose d'abord sur les modes de fonctionnement de la revue. Elle est née de l'initiative de jeunes chercheurs et ce sont eux aussi qui en assurent la 
dynamique de fond. L'essentiel du travail de rédaction s'effectue au sein d'un comité de rédaction constitué de doctorants et de post-doctorants issus du CIERA et renouvelé régulièrement pour préserver l'idée initiale d'une revue faite par et pour des jeunes chercheurs. Ce comité est entièrement responsable de la ligne éditoriale (par exemple la définition des rubriques, le choix des thématiques retenues pour les dossiers) et de la qualité scientifique des textes publiés (lancement d'appels à communication, choix des articles, encadrement des auteurs). Or il n'est pas rare que cette responsabilité se traduise par un travail approfondi d'assistance auprès des jeunes auteurs pour lesquels un article dansTr@jectoires constitue bien souvent leur première publication. La revue remplit alors une triple fonction essentielle :

1. initier des jeunes chercheurs aux règles de la rédaction scientifique et aux exigences auxquelles se doit de répondre, aujourd'hui, une publication de qualité ;

2. permettre aux jeunes chercheurs, pour lesquels l'accès aux « grandes » revues scientifiques reste souvent un obstacle, de prendre la parole et de participer aux réflexions et aux débats de la recherche franco-allemande en sciences humaines et sociales ;

3. offrir à des doctorants avancés et aux jeunes chercheurs "confirmés » la possibilité, en faisant partie du comité, de bénéficier d'une première expérience éditoriale et donc d'apprendre la gestion d'un instrument fondamental de la production et de la diffusion des savoirs scientifiques.

3 Si publier un article dans Tr@jectoires représente, par rapport aux contraintes imposées par une revue "classique ", une charge moins lourde (les articles tournent autour d'une dizaine de pages), la revue n'en offre pas moins tous les gages d'un examen critique des manuscrits et l'occasion de découvrir l'évaluation par les pairs. Dans ce travail, la rédaction est d'ailleurs soutenue par le comité de pilotage du CIERA, composé d'enseignants-chercheurs représentant les établissements membres du Centre. Ce comité porte une attention constante à la meilleure qualité scientifique possible de la revue dans son ensemble.

\section{Les rubriques}

4 La revue s'organise en quatre rubriques principales, toutes placées dans un horizon franco-allemand (que ces approches soient comparatives ou non) et au croisement des époques et des disciplines. Elle comporte d'abord un Dossier thématique dont le sujet varie au fil des numéros. S'articulant autour d'un thème de recherche commun choisi pour son actualité et sa pertinence scientifiques, ce dossier vise à refléter les questions et les débats soulevés par des travaux en cours. À l'heure actuelle, trois thèmes ont déjà été traités : en 2007 les questions liées au genre (dossier « masculin/féminin », coordonné par C. Chamayou-Kuhn, P. Farges et P.E. Yavuz), en 2008 les ambiguïtés et la dynamique des frontières (dossier "frontières en question", coordonné par C. Chamayou-Kuhn, S. Iglesias, C. Quéva, A. Richter et Y. Vilain) et, en préparation pour 2009, le renouveau de la narration en sciences humaines et sociales (dossier "mondes en narration", coordonné par M. Berdet, T. Chaix-Bryan, C. Chamayou-Kuhn, M. Dubois, S. Kellerer, P. Krilles, A. Kwaschik, A. Richter et P.E. Yavuz).

5 Le dossier thématique de chaque numéro est ensuite complété par la rubrique Perspectives qui intègre des articles présentés pour leur intérêt scientifique et l'originalité du sujet abordé. Cette partie est par conséquent marquée par une plus grande ouverture thématique. Elle a pour objectif de montrer toute la richesse de la jeune recherche 
franco-allemande et elle se prête en outre particulièrement bien à une première expérience de publication permettant à des auteurs néophytes de s'habituer, avec l'aide du comité de rédaction, aux impératifs de l'écriture scientifique. L'éventail des textes publiés depuis 2007 dans cette rubrique est large, associant la variété disciplinaire à la diversité thématique et mettant notamment en exergue la pluralité des approches méthodologiques.

6 La troisième rubrique de la revue rassemble, sous le nom de SynTHÈSES, les résumés des dernières thèses soutenues par les inscrits du CIERA. À l'instar du Bulletin d'Information de la MHFA, Tr@jectoires propose ainsi aux jeunes docteurs une vitrine pour faire connaître, auprès d'anciens et de futurs collègues, les résultats de leurs recherches dans leur ensemble. Généralement, les auteurs optent pour un résumé de thèse en français quand la thèse a été écrite en allemand, et vice-versa. La revue contribue par là, à sa façon, à jeter un pont entre les deux mondes universitaires français et allemand afin de faciliter, à l'intérieur d'un espace européen de la recherche, la circulation des idées et une meilleure connaissance des chercheurs. Dans la même perspective, chaque article publié par la revue est accompagné de deux résumés (en français et en allemand).

Quatrième rubrique, enfin, la CIERAthèque propose une liste des dernières publications des jeunes chercheurs du CIERA. En outre, la revue publie le compte-rendu des travaux effectués lors du séminaire franco-allemand de jeunes chercheurs que le CIERA organise chaque année au Moulin d'Andé.

\section{Sur la Toile mondiale...}

8 En dehors de son fonctionnement et de ses orientations éditoriales, Tr@jectoires se distingue également par sa forme. D'emblée, la revue a été pensée comme un périodique électronique. Publiée, dans un premier temps, en libre accès sur le site web du CIERA, elle a néanmoins donné lieu à l'édition papier des deux premiers numéros, notamment pour faciliter sa distribution et rendre son existence plus concrète. Même si cette possibilité d'une double édition ne sera pas abandonnée définitivement, la revue va franchir bientôt un prochain seuil en intégrant, sous le nom de Trajectoires, le portail revues.org autrement dit la plus importante plate-forme française de revues en sciences humaines et sociales (avec plus d'un million de visiteurs par mois). Répondant aux transformations actuelles de l'édition scientifique, la revue gagnera ainsi amplement en visibilité. Au-delà du périmètre franco-allemand, son rayonnement sera désormais international. De surcroît, son acceptation par le comité scientifique de revues.org marque une reconnaissance du travail effectué jusqu'ici par le comité de rédaction et de la pertinence des textes déjà parus, le portail n'accueillant que des revues dotées de procédures qui garantissent la qualité des contributions publiées. Ces évolutions incitent donc à espérer que Trajectoires continuera à honorer son rôle de seule revue franco-allemande qui propose à la jeune recherche en sciences humaines et sociales un forum de qualité, ainsi qu'un outil adapté au besoin qu'elle a d'être visible et de valoriser ses travaux.

$9 \quad$ Tr@jectoires. Travaux des jeunes chercheurs du CIERA

CIERA

Maison de la recherche, 28 rue Serpente, 75006 Paris

Tél. : (00-33) (0)1 53105736

Fax : (00-33) (0)153105739

Courriel : trajectoires@ciera.fr 
Internet : http://www.ciera.fr/ciera/spip.php?rubrique118

Responsables : Elissa Mailänder-Koslov (responsable de la publication), Annette Schläfer (réalisation technique)

\section{NOTES}

1. Le CIERA, crée en 2001 et situé à Paris, rassemble en réseau onze établissements français d'enseignement supérieur et de recherche. Il est soutenu par le DAAD (Deutscher Akademischer Austauschdienst) et le Ministère de l'Enseignement supérieur et de la Recherche.

\section{AUTEUR}

\section{FALK BRETSCHNEIDER}

Falk Bretschneider est maître de conférences à l'École des hautes études en sciences sociales à Paris. De 2006 à 2008, il a coordonné le programme d'encadrement doctoral du CIERA et il est cofondateur de la revue Tr@jectoires. 